\title{
Contrast Effects In Verbal Output
}

\author{
Melvin Manis and Gregory W. Armstrong \\ University of Michigan and Ann Arbor Veterans Administration Hospital ${ }^{1}$
}

\begin{abstract}
Ss' freely constructed descriptions of several photographs, showing posed emotional expressions, were partly dependent on the array of contextual stimuli (other photographs) that appeared on the same page. Ss who were assigned to a predominantly pleasant array of photographs produced the least pleasant descriptive passages, followed by those in an unbiased (full range) condition, and finally, those $S \mathrm{~s}$ who were assigned to an unpleasant context, whose descriptions were the most pleasant of all three groups. This experiment suggests that when A communicates with B concerning some external referent, the meaning that he conveys may be significantly affected by the context surrounding his intended referent.
\end{abstract}

Studies of absolute judgment have repeatedly shown that the response elicited by a given stimulus may be affected not only by the characteristics of that stimulus, but by the total array of stimuli to which the $S$ has been exposed (Helson, 1964; Bevan, 1968). The contrast effect is perhaps the best known of these phenomena. An experiment by Campbell, Hunt, and Lewis (1958) is typical of many studies in this domain. These investigators demonstrated that in assessing the disorganization and eccentricity of thought implied by different vocabulary definitions, judges who had been given a large number of "high-pathology" definitions tended to rate midscale items as indicating less pathology than did judges who were given a predominance of "low-pathology" definitions. That is, there was an apparent contrast between the overall array of stimuli presented to the individual judge, and his response to the midscale "test" items.

A study by Manis (1967) demonstrated the applicability of the contrast principle within a communication setting. The subjects in this experiment were presented with a series of written passages, each describing an actor's portrayal of some emotional state. Ss were also given the 11 photographs that had been used to elicit these descrip-

${ }^{1}$ All statements are those of the authors and do not necessarily represent the opinions or policy of the Veterans Administration. This research was also supported in part by NSF Grant GS-1116.

(C) 1971 by Academic Press, Inc. 
tions, and wcre instructed to indicate the picture (referent) that was described in each passage. Some $S$ s were given descriptions of emotions that had previously been rated as predominantly pleasant (Schlosberg, 1952); others were given mainly unpleasant descriptions, and a third group responded to an unbiased series containing both pleasant and unpleasant descriptions. The results showed clearcut evidence of contrast. Ss assigned to the unpleasant context selected relatively pleasant photographs as the referents for a set of neutral "test" descriptions, while those assigned to the pleasant context selected relatively unpleasant photographs in response to these passages; $S$ s who had read the unbiased series of descriptions generally responded to the test descriptions by choosing referents that fell between these two extreme groups.

The present experiment was designed to provide additional information regarding the impact of the contrast phenomenon on the communication process. In this case, however, rather than focussing attention on the behavior of the message recipient (as in Manis, 1967), our primary concern was centered on the verbal output of the message sender. Briefly stated, the S's task was to construct a series of written messages that described various referents (photographs of posed emotional expressions). Descriptions were generated under varying contextual conditions. More particularly, this experiment compared descriptive passages that were written by $S$ s who had been exposed to contrasting arrays of potential referents (e.g., photographs of predominantly pleasant or unpleasant emotional expressions), but who had been instructed to write about the same subsample of test referents (photographs). If the overall array mainly included pleasant emotional expressions, the contrast hypothesis suggests that a test photograph depicting a neutral emotion should be described in relatively unpleasant terms; on the other hand, a $S$ assigned to the unpleasant context should produce relatively pleasant test messages.

\section{METHOD}

Sixty Ss at the University of Michigan were recruited as paid subjects for an experiment concerned with communication. They were randomly divided into three main groups, each containing $20 \mathrm{Ss}$. Each $S$ was presented with an $8 \times 10$ sheet containing either 16 or 24 randomly arranged photographs from the Frois-Wittman series of posed emotional expressions.

The Ss were asked to "spend a few seconds looking at each picture and thinking very briefly how you might describe it." They were then instructed to write passages describing either 8 or 12 selected photographs from their respective picture arrays. In each case, the writer was to give his impression of the "mood, feelings, or thoughts of the person pictured," or to describe the "situation he is in and his reaction to it." "The writers were encouraged to "make each description clear 
cnough so that somcone clse would know which picture you've written about." The following passages are reasonably typical of those that were produced:

"Someone is going to hit him or is menacing him, and he is pleading with the fellow to talk things over. Ile is concerned about his personal safety, but terribly desperate."

"He is inwardly chuckling at a joke or a 'boner' that someone has pulled. Rather than laugh openly, he just makes a half-hearted mouth gesture to himself or maybe to another person who will see the humor the same way he does."

"The man is deeply involved in watching a James Bond movie. The hero is in trouble again, and the man doesn't dare take his eyes off Bond for fear he'll miss something."

The diverse arrays of photographs. There were three different arrays of emotional expressions, each of which included a common set of eight core photographs depicting neutral expressions. The core photographs, in turn, consisted of two parallel test sets, each containing four photographs that were matched in terms of their pleasantness ratings (Schlosberg, 1952). The two test sets (set A and set B) were assembled to check on the generality of any effects that might be obtained. Half the $S s$ assigned to a given array $X$ context strength combination (e.g., pleasant array, strong context-see below) wrote test passages describing neutral test photographs from set $A$, while the other half wrote about the test photographs in set $B$.

The three picture arrays in which the neutral test photos were embedded are described below:

1. The Plesant array of 16 photographs included 8 relatively pleasant emotions, in addition to the common core of 8 neutrals. All $S_{s}$ in this condition described 8 photographs, as detailed below.

2. The Unpleasant array also included 16 photographs; 8 that depicted unpleasant emotions, plus the 8 neutral photographs of the core set. Ss assigned to the unpleasant array described 8 photographs.

3. The Full Range array consisted of 24 photographs that spanned the entire pleasantness continuum as calibrated in Schlosberg's norms (1952). Ss assigned to the Weak Context version of the full array (see below) described 8 photographs; those assigned to the Strong Context, full array, described 12 photographs.

Strength of context. Half of the subjects described only the neutral (core) photographs that appeared on all of the picture sheets. This was termed the Weak Context condition, for the photographs that determined the distinctiveness of the various arrays were simply "present," to be viewed passively. To control for the total number of passages written by each $S$, those who were assigned to the Weak Context condition were instructed to describe the photographs in both test sets. Among weak context subjects who were assigned to test set A, however, the descriptions of the set B pictures were not included in the final data analysis; similarly the set A descriptions were disregarded for those $S$ s assigned to test set $B$.

$S_{s}$ in the Strong Context condition described not only the four neutral photographs in their assigned test set (A or B), but also produced several passages based on the context photographs that were included on their picture sheets. In planning this aspect of the experiment, it was anticipated that displacement effects might be particularly prominent when the $S$ s were required to deal actively with the background photographs, as they did in the strong context conditions.

Trest and context ariting trials. Writers in the Full Range, Strong Contest con- 
dition described eight context photographs within a total series of 12 writing trials; their test passages were written on trials $5,8,11$, and 12 , while context passages (four pleasant and four unpleasant) were written on the remaining trials. Ss assigned to all other experimental groups wrote four "nontest" passages within a total series of eight writing trials, with the test descriptions being produced on trials $3,5,7$, and 8 .

Rating the messages. The passages produced by the experimental Ss (writers) were converted into a common quantitative system by means of a rating procedure. To provide the judges with a stable and unbiased context, each rating booklet contained passages describing the full range of emotional expression (including some passages that had been collected for another study). Each booklet included 108 descriptions based on neutral photographs, which were randomly mixed with 54 pleasant and 54 unpleasant descriptions (these latter passages had been obtained from writers in the strong context conditions). Test descriptions from the various experimental conditions were scattered widely throughout each booklet to avoid any systematic order effects in the ratings that were assigned. In assembling the booklets, descriptions that were based on any single photograph were placed in nonadjacent positions; moreover, an effort was made to avoid unintended context effects (as might occur if, for example, the test descriptions from one writing condition were commonly preceded by descriplive passages based on unpleasant poses).

The raters used a 9-point scale, ranging from -4 to +4 , to indicate the "pleasantness-unpleasantness of the emotional state of the actor in each description." These ratings were later transformed into a simple $1-9$ scale. Katers were to "avoid considerations ... of a purely grammatical or stylistic nature," and were simply to give their "judgment of the pleasantness-unpleasantness of the emotional experience described." The judges were not given any information regarding the diverse experimental conditions under which the messages had been produced. Each test passage was rated by four different judges, although a total of 20 different raters participated in this phase of the study. The dependent variable of the experiment was the mean rating elicited by each test passage.

Interrater reliability was assessed by correlating the ratings of various pairs of judges across a common subset of 48 neutral descriptions; the sample of descriptions that was used for this purpose included test messages that were generated in each of the experimental conditions represented in the study. The results indicated that there was satisfactory reliability, for interjudge correlations of $.61, .67$, and .88 were obtained.

\section{RESULTS}

The data were analyzed in a complex analysis of variance based on three between-subject variables and one variable within-subjects. The between-subjects factors were: $(a)$ the three arrays of photographs, (b) context strength (strong versus weak), and (c) the particular set of test photographs that had been assigned to the $S$ ( set A versus set B). The within-subject variable was based on the rated pleasantness of the four photographs in each test set (Schlosberg, 1952).

As shown in Table 1 , the three picture arrays led to significantly different test passages. The obtained pattern of results was consistent 
with theoretical expectations for there was an inverse relationship between the pleasantness of photographs in the three arrays, and the rated pleasantness of the resulting test messages. That is, $S_{\mathrm{s}}$ assigned to the pleasant array produced the least pleasant test messages (mean rating $=4.1$ ), followed by the full range group (mcan rating $=4.7$ ), and finally the $S$ s assigned to the unpleasant array of photographs, whose descriptions were judged to be most pleasant of all (mean rating $=5.1$ ).

While the same gross pattern of results was obtained with both sets of test photographs ( A and B), the contrast effect was stronger for Ss assigned to set $\mathrm{A}$, as reflected in the significant $\mathrm{A} \times \mathrm{C}$ interaction. This result suggests that despite the replicability of the contrast phenomenon, sets of photographs that are matched on the basis of pleasantness values may nevertheless differ significantly in their susceptibility to contextual influences.

Table 1 shows several other significant effects, although these are of limited theoretical interest. There were, for example, significant effects

TABLF 1

Summary of AN.ILYis OF VARIANCE

\begin{tabular}{|c|c|c|c|}
\hline Source & $d f$ & $M S$ & $F$ \\
\hline Between subjects & 59 & & \\
\hline Context (A) & 2 & 17.651 & $12.51^{* * *}$ \\
\hline Context strength (B) & 1 & 1.998 & \\
\hline Test set. $(C)$ & 1 & 0.392 & \\
\hline$A \times B$ & 2 & $: 3.296$ & \\
\hline $\mathrm{A} \times \mathrm{C}$ & 2 & 5.923 & $4.20^{* *}$ \\
\hline $\mathrm{B} \times \mathrm{C}$ & 1 & 2.501 & \\
\hline $\mathrm{A} \times \mathrm{B} \times \mathrm{C}$ & 2 & 0.729 & \\
\hline Ss within groups & 48 & 1.411 & \\
\hline Within subjects & 180 & & \\
\hline Photograph value (1)) & 3 & 56.458 & $38.96^{* * *}$ \\
\hline$A \times 1)$ & 6 & 2.364 & \\
\hline$B \times 1)$ & 3 & 1.507 & \\
\hline $\mathrm{C} \times \mathrm{D}$ & 3 & 9.206 & $6.35^{* * *}$ \\
\hline $\mathbf{A} \times \mathbf{B} \times 1)$ & 6 & 3.489 & $2.41^{*}$ \\
\hline $\mathrm{A} \times \mathrm{C} \times \mathrm{D}$ & 6 & 2.325 & \\
\hline $\mathrm{B} \times \mathrm{C} \times 1)$ & 3 & 1.011 & \\
\hline $\mathrm{A} \times \mathrm{B} \times \mathrm{C} \times \mathrm{D}$ & 6 & 1.350 & \\
\hline D $\times$ Ss within groups & 144 & 1.449 & \\
\hline Total & 239 & & \\
\hline
\end{tabular}

$* p<.05$

${ }^{* *} p<.025$.

$* * * p<.001$. 
associated with the pleasantness of the test photographs (variable D); this simply means that the more pleasant test photographs (as calibrated in Schlosberg's norms) led to descriptive passages that were rated as relatively pleasant. The significant $\mathrm{C} \times \mathrm{D}$ interaction, between the two sets of test photographs and the pleasantness ratings of the individual pictures, means that the two test sets were not perfectly matched. That is, when data from the various context groups were combined, the differences between the passages based on individual photographs in test set $A$, were not paralleled by the differences obtained in response to set $\mathrm{B}$.

The significant $\mathrm{A} \times \mathrm{B} \times \mathrm{D}$ interaction (photograph array $\times$ context strength $X$ pleasantness value of the test photograph) defies any confident interpretation at the present time. The complexity of this interaction, together with the absence of any convincing theoretical explanation for its appearance, suggest that it may be of doubtful replicability. Apart from its contribution to this unexpected interaction, the context strength variable did not significantly affect the $S s^{\prime}$ verbal output; our effort to enhance the potency of the various referent arrays by manipulating the $S$ 's active attention through variations in his writing assignment was apparently unsuccessful.

The fact that similar results were obtained in the weak and strong context conditions was unexpected. However, the ineffectiveness of this variable permits us to reject what night be termed an "equal frequency" interpretation for the present results. Recall that the $S_{S}$ in the weak context groups did not differ in the test photographs that they described, although they did differ in the arrays of photographs that they saw. Nevertheless, the messages produced by these writers showed clear evidence of contrast. This means that the systematic displacements observed in the weak context condition cannot readily be attributed to the Ss' implicit attempts to produce roughly equal frequencies of pleasant and unpleasant descriptions.

\section{DISCUSSION}

This experiment testifies to the significance of contextual stimuli as determinants of natural language behavior, a phenomenon of obvious importance and generality. The results indicate that when A speaks to $B$ concerning some external referent, the meaning that he conveys may be significantly affected by the context to which he has been exposed.

The underlying mechanism for this effect is somewhat unclear, however. One line of analysis (e.g., Helson, 1964) suggests that the phenomenon is primarily perceptual in nature. In the present experiments, this would imply that the variations in context were instrumental in 
affecting the subjective impressions that the various photographs evoked, which in turn were reflected in the writer's descriptions. Thus, when embedded in an array of pleasant photographs, a neutral picture might simply look less pleasant than it would under uther circumstances and would, as a result, be described in relatively unpleasant terms.

An alternative explanation would emphasize the context as a direct determinant of the writer's descriptions. Explanations of this type are basically semantic in character, for they focus on the labeling systems that are utilized under divergent contextual circumstances. A welldeveloped theory of this sort, with particular relcvance for the present experiment, may be found in Rosenberg and Cohen's analysis (1966) of the referential process (also see Rosenberg \& Gordon, 1968). These theorists suggest that when a speaker is faced with the task of describing a given referent, the message that he ultimately generates will be partly determined by a comparison process, which leads him to inhibit certain descriptions that have implicitly been evoked, but must be rejected as unsuitable because they are associated both with the speaker's intended referent, and with various nonreferents with which it might be confused. According to this view, contextual influences may affect the writer's overt description by inducing him to inhibit verbalizations that might be frecly emitted in other circumstances.

The Rosenberg-Cohen account can be applied to this experiment by noting that the average $S$ would presumably avoid descriptive comments which might be relevant to several of the photographs in his assigned array. For example, given a predominantly pleasant set of photographs, a writer might conclude that a neutral test picture could best be distinguished from the others in the array by emphasizing its unpleasant aspects. This hypothesized selection process seems particularly plausible in view of the instruction that each description be "clear enough that someone else would know which picture you've written about."

On the other hand, the Rosenberg-Cohen approach may be less directly applicable to an experiment which was subsequently conducted in this laboratory. ${ }^{2}$ In this study contrast effects were produced by having $S_{s}$ read written descriptions of various pleasant (or unpleasant) emotions, during a session in which they intermittently wrote their own descriptions of several posed emotions. The writers were simply asked to describe the different test photographs. Nothing was said about the desirability of generating passages that would permit a clear discrimination between the emotions that the writers read about (the context),

" This experiment was performed by Stephanie Riger. 
and those that scrved as referents for their own descriptions (the test photographs). Despite this, the resulting passages were systematically contrasted "away from" the messages that the writers had read. The Rosenberg-Cohen theory may apply here as well, however, if we emphasize the possibility that in screening implicit verbalizations that have been stimulated by his assigned referent, the writer may be significantly affected by the salience of various nonreferents that operate at a purely symbolic level. Thus, by having the writer read about different emotional expressions, we may strengthen his awareness ("remind" him) of certain nonreferent emotions, and induce him to inhibit ambiguous descriptions that might otherwise be freely emitted.

The present results provide additional evidence concerning the relevance of the contrast phenomenon for the study of communication. Contrast effects appear to reflect a very general behavioral principle that plays a significant role in communication and presumably in other cognitive activities as well.

\section{REFERENCES}

Bevan, W. Contextual basis of behavior American Psychologist, 1968, 23, 701-714.

Camprell, D. T., Hunt, W. A., \& Lewis, N. A. The relative susceptibility of two rating scales to disturbance resulting from shifts in stimulus context. Journal of Applied Psychology, 1958, 42, 213-217.

Helson, H. Adaptation-level theory: An experimental and systematic approach to behavior. New York: Harper \& Row, 1964.

Manis, M. Context effects in communication. Journal of Personality and Social Psychology, 1967, 5, 326-334.

Rosenberg, S., \& Cohen, B. D. Referential processes of speakers and listeners. Psychological Review, 1966, 73, 208-231.

Rosenberg, S., \& Gordon, A. Identification of facial expressions from affective descriptions: a probabilistic choice analysis of referential ambiguity. Journal of Personality and Social Psychology, 1968, 10, 157-166.

Schlosberg, $H$. The description of facial expressions in terms of two dimensions. Journal of Experimental Psychology, 1952, 44, 229-237.

(Received April 15, 1970) 